\title{
ESTRUCTURA SOCIAL DEL DELFÍN NARÍZ DE BOTELLA Tursiops truncatus (CETACEA: DELPHINIDAE) EN LA COSTA SUROESTE DE LA ISLA DE TENERIFE (ISLAS CANARIAS), ESPAÑA
}

\section{SOCIAL STRUCTURE OF THE BOTTLENOSE DOLPHIN Tursiops truncatus (CETACEA: DELPHINIDAE) IN THE SOUTHEASTERN COAST OF TENERIFE ISLAND (CANARY ISLANDS), SPAIN}

\author{
Valeria Verme ${ }^{1}$ y José Iannacone ${ }^{1,2}$
}

\begin{abstract}
Resumen
El delfín nariz de botella Tursiops truncatus juega un rol muy importante en la industria de avistamiento de cetáceos en la isla de Tenerife (Islas Canarias), España. El objetivo de este trabajo fue determinar la estructura social de $T$. truncatus en la costa suroeste de Tenerife para los años 2005 y 2006, en un área de estudio de $180 \mathrm{~km}^{2}$. Se foto-identificaron 129 delfines, 70 de los cuales se consideran residentes (54,3\%), porque se observaron en ambos años del estudio. La población estimada en el área de estudio fue de 143 delfines nariz de botella, con una densidad de 0,79 delfines $\cdot \mathrm{km}^{-2}$. El número de delfines por grupo varió entre 2 y 10 individuos, aunque ocasionalmente se observaron grupos de hasta 50 delfines. Los meses de presencia de crías fue entre junio y noviembre con un pico en agosto (verano). Se determinó que hay asociaciones fuertes entre hembras avistadas juntas. El índice de asociación (HWI) mostró valores entre 0,09 a 0,30.
\end{abstract}

Palabras clave: avistamiento de cetáceo, cría, estructura social, foto-identificación, grado de residencia.

\begin{abstract}
The bottlenose dolphin Tursiops truncatus (Montagu, 1821) plays an important role in the whale watching industry in the island of Tenerife (Canary Islands), Spain. The aim of this study was to determine the social structure of T. truncatus on the South Eastern coast of Tenerife for the years 2005 and 2006, in a study area of $180 \mathrm{~km}^{2} .129$ dolphins were photo-identified, 70 of which were considered as residents (54.3\%), because they were observed both years. The estimated population in the study area was 143 bottlenose dolphins, with a density of 0.79 dolphins $\mathrm{km}^{-2}$. The number of dolphins per group varied from 2 to 10 individuals. Groups comprised of more than 50 dolphins have been sighted, although this was not very common. Offspring were observed from June to November, with the peak in August (summer). It was determined that there are strong associations between female dolphins sighted together. Index of association (HWI) showed values from 0,09 to 0,30 .
\end{abstract}

Key words: cetaceans, degree of residence, offspring, photo-identification, social structure.

\section{Introducción.}

El delfín nariz de botella o tonina Tursiops truncatus (Montagu, 1821) (Cetacea: Delphinidae) es la especie de cetáceo más conocida por su distribución cosmopolita (Aguayo-Lobo, 1999) y su gran adaptabilidad a ambientes artificiales como delfinarios y zoológicos alrededor del mundo (Folkens et al., 2002; Bejder et al., 2006; ACS, 2007; CITES, 2007), lo cual facilita su estudio en estado silvestre y en cautiverio (Bearzi, 2005; WDCS, 2007). Hoy día se reconocen dos especies, el "delfín nariz de botella” del indo-pacífico Tursiops aduncus (Ehrenberg, 1833), el cual habita cerca de Australia, en el Océano Indico, China y Sud África y el delfín nariz de botella $T$. truncatus con una distribución cosmopolita que incluye aguas templadas y tropicales (Waerebeek et al., 1997; Bearzi, 2005; May-Collado et al., 2007; Fury \& Harrison, 2008; Genow et al., 2008; Foley et al., 2010). Además se reconocen dos ecotipos del delfín nariz de botella, uno oceánico y otro costero (Vázquez et al., 2009). El ecotipo costero habita aguas cálidas y de poca profundidad y son típicamente más pequeñas que el ecotipo oceánico. Además, tienen una pigmentación más clara y las aletas son proporcionalmente más grandes que los individuos que viven en aguas oceánicas (Eisfeld, 2003; Vázquez et al., 2009). Contrario al ecotipo oceánico el cual forma grupos grandes de hasta cientos de individuos, los delfines costeros se observan en grupos pequeños de 2 a 15 individuos en zonas costeras (Majluf \& Reyes, 1989; Lodi et al., 2008; Pérez-Cao et al., 2009). 
Los delfines nariz de botella tienen una sociedad fisión-fusión, en donde la composición y estabilidad del grupo varía a lo largo del día (Vázquez et al., 2009; Foley et al., 2010; Randic et al., 2012). Por lo general los machos son los que entran y salen de los grupos, aunque en algunas sociedades estos pueden formar alianzas de hasta más de 20 años, mientras que, las hembras pueden mantenerse en el grupo en forma más permanente (Folkens et al., 2002; MartínezSerrano et al., 2011; Randic et al., 2012). En Australia, los delfines nariz de botella pueden formar dos niveles distintos de alianzas, conformando redes sociales de hasta 400 individuos (Foley et al., 2010; Martínez-Serrano et al., 2011; Lodi \& Monteiro-Neto, 2012). Este comportamiento no se había registrado en otra especie de mamífero con excepción del Papio cynocephalus (Linnaeus, 1766) "babuinos" con una intrincada organización social (Connor et al., 1999).

A pesar de que el delfín nariz de botella se considera la especie más avistada de las Islas Canarias, son escasos los estudios en el área (Plasencia et al., 2001; Navarro, 2002; Jaber et al., 2005; Torres et al., 2005; Bearzi et al., 2008; Carballo et al., 2008; Esperón et al., 2008; Pérez-Vallazza et al., 2008; Carrillo et al., 2010; CC, 2010; Verme \& Iannacone, 2011), El objetivo de este estudio es determinar la estructura social de la población del delfín nariz de botella utilizando la técnica de fotoidentificación, un método de captura-recaptura que permite identificar individuos con base a marcas naturales y permanentes en sus aletas dorsales (Verme \& Iannacone, 2011). Además proveemos las primeras estimaciones de tamaño poblacional, residencia, composición y tamaño del grupo, así como patrones de asociación entre delfines utilizando datos colectados en los años 2005 y 2006.

\section{Materiales y métodos.}

Zona de estudio.

Se realizó en la zona sur oeste de la isla Tenerife, que forma parte del archipiélago de las Islas Canarias en el Océano Atlántico. El área comprende desde la bahía "Masca” (1652'00” LW/ 28¹7’20” LN) hasta

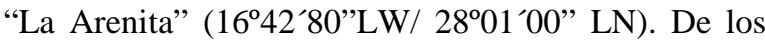
tres puertos principales de norte a sur: Los Gigantes (16 50'50” LW/ 28 04'70” LN), Puerto Colón (16 $44^{\prime} 20^{\prime \prime}$ LW/ 28 $04^{\prime} 50^{\prime \prime}$ LN) y Los Cristianos (16 $\left.43^{\circ} 00^{\prime \prime} \mathrm{LW} / 2^{\circ} 02^{\prime} 70^{\prime \prime} \mathrm{LN}\right)$ salen las embarcaciones turísticas para el avistamiento de los cetáceos (Observar mapa del área de estudio en Verme \& Iannacone, 2011). En estas aguas marinas se han avistado un total de 26 diferentes especies de cetáceos, entre misticetos (ballenas con barbas) y odontocetos (ballenas dentadas) (Vonk \& Martel, 1988). En ciertas zonas del fondo marino se han observado praderas de algas de Cymodocea nodosa (Ascherson 1869), las que están protegidas según la normatividad de las islas Canarias (España), debido a que evitan la erosión del suelo marino y son el alimento primordial de muchas especies de peces y tortugas marinas. También encontramos corales entre los 50 y $600 \mathrm{~m}$ de profundidad, albergando diversas especies características de este ecosistema. Entre las especies pelágico costeras tenemos al pejerrey (Atherina), la boga (Boops) y la palometa (Trachinotus). En aguas abiertas, tenemos peces como la caballa (Scomber) o la sardina (Sardinella aurita Valenciennes in Cuvier and Valenciennes, 1847), que se acercan a las costas para su reproducción y el desarrollo de su fase juvenil. Data recopilada de los delfines.

Se recopiló desde mayo 2005 a noviembre 2006. Las estaciones fueron definidas como: primavera (abril a junio), verano (julio a septiembre), otoño (octubre a diciembre) e invierno (enero a marzo). Para la toma de los datos se requirió la presencia de voluntarios de la Atlantic Whale Foundation (AWF) por lo que el esfuerzo de la toma de datos no fue homogéneo a lo largo de ambos años. Existieron meses en los que no se obtuvieron información de avistamientos. Las fotografías se tomaron a bordo de doce barcos ecoturísticos de avistamiento de cetáceos que salen de los tres puertos principales. Las embarcaciones a motor variaron en tipo y tamaño (Catamaranes de $35 \mathrm{~m}$ de eslora, y veleros de 12 y 20 $m$ de eslora). Las horas en alta mar fluctuaron entre 2 a 6, a velocidades entre 15 a $20 \mathrm{~km} \cdot \mathrm{h}$ (MartínezSerrano et al., 2011).

Una vez observados los delfines se disminuyó la velocidad y la embarcación se acercaba de forma paralela hasta una distancia promedio entre 20 y $60 \mathrm{~m}$. El empleo del término avistamiento siguió a MartínezSerrano et al. (2011). Un avistamiento se refiere a la observación de uno o más delfines realizado por un voluntario desde una embarcación, empleándose un formulario para cada caso (Verme \& Iannacone, 2011). A veces los delfines se aproximaban a las embarcaciones y nadaban en la proa ("bow riding”). Siempre se trató de no perturbar a los delfines, evitando que la embarcación divida al grupo de delfines que ingresaban al medio, o se maniobró para acercarse al grupo por detrás para no perturbar su comportamiento natural. Se empleó un total de 70,1 h de interacción con los delfines. Para la fotoidentificación de las marcas de la aleta dorsal de los delfines nariz de botella se siguió el procedimiento estándar descrito en detalle por Grellier et al. (2003), y Verme \& Iannacone (2011) (Figura 1). También se consideraron marcas en otras partes del cuerpo que ayudaban a la identificación individual (Figura 2). Las fotografías fueron tomadas con cámaras digitales Nikon D50 con zoom de $18-70 \mathrm{~mm}$. En total se tomaron más de 3000 fotografías durante el 2005 y 2006. Junto con las fotografías se llenó un formulario general con datos como: 1) nombre de la embarcación; 2) hora de inicio y fin de la interacción; 3) velocidad de la embarcación en el momento de la interacción; 4) 


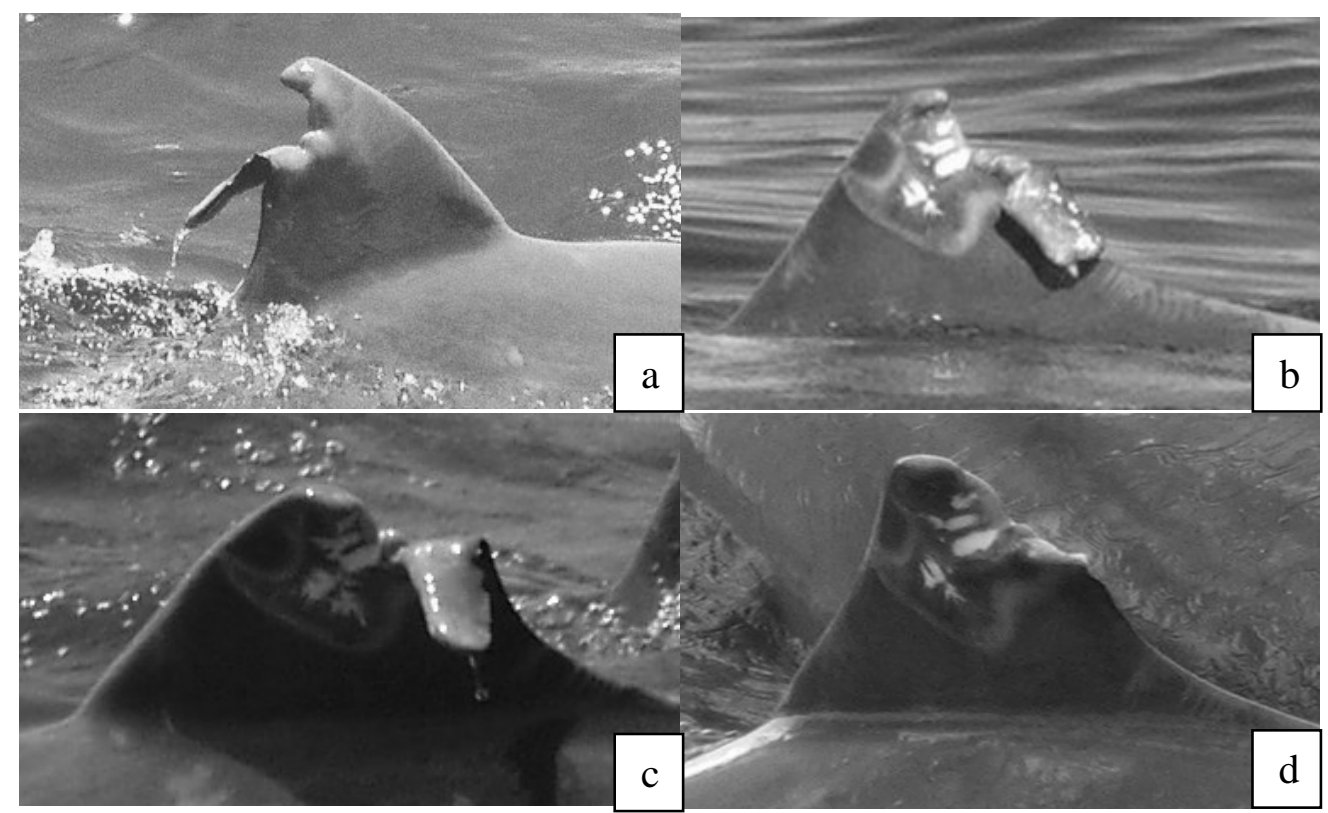

Figura 1. Secuencia de fotografías del cambio progresivo de la herida de uno de los delfines en la aleta dorsal. a. 19.07.05. b. 16.08.05. c. 16.11.05. d. 30.11.05.

estado del mar; 5) estado del tiempo; 6) posición global; 7) número de individuos avistados; 8) identificación de adultos y crías, y 9) presencia de otras embarcaciones. La selección de las fotografías fue realizada según lo señalado por Verme \& Iannacone (2011).

Residencia.

Los animales residentes fueron aquellos que estuvieron presentes en ambos años indicando alta fidelidad en la zona de estudio (Rossi-Santos et al., 2007), durante el 2005 y el 2006. Se siguió a Martínez-Serrano et al. (2011) para la clasificación de los delfines en residentes y visitantes.

Tamaño poblacional.

La estimación del tamaño de la población de delfines en el área de estudio se obtuvo del modelo captura-recaptura en base a la fórmula de Petersen modificado por Bailey, en la que cualquier individuo de la población tiene la misma probabilidad de ser censado (Felix, 1994):

$$
\mathrm{N}=\mathrm{n}_{1}\left(\mathrm{n}_{2}+1\right) / \mathrm{m}_{2}+1
$$

Donde $\mathrm{N}$ es el tamaño de la población, $\mathrm{n}_{1}$ es el número de individuos identificados en el 2005, $\mathrm{n}_{2}$ es el número de individuos identificados en el $2006 \mathrm{y} \mathrm{m}_{2}$ es el número de individuos avistados en ambos años. Para evaluar el grado de similaridad entre ambos años, se utilizaron tres índices cualitativos en base a los individuos identificados: Jaccard $\left(\mathrm{I}_{\mathrm{j}}\right)$, Sörensen $\left(\mathrm{I}_{\mathrm{s}}\right)$ y Ochiai-Barkman $\left(\mathrm{I}_{\mathrm{O}-\mathrm{B}}\right)$, los cuales muestran alta similaridad al tender el valor a 1 y baja similaridad con valores tendientes a 0 (Moreno, 2001).
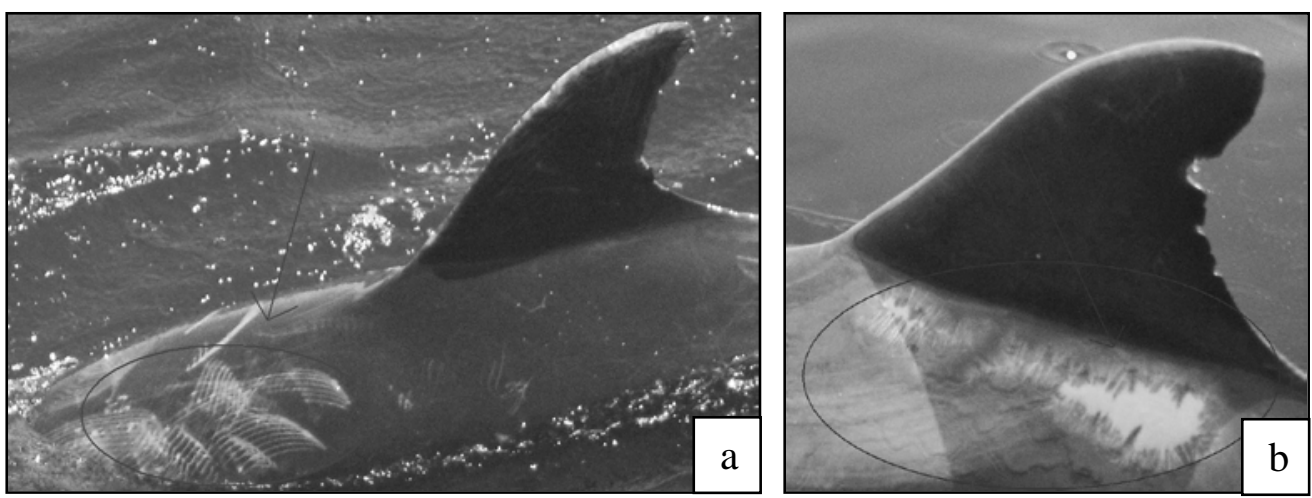

Figura 2. Delfín T. truncatus con a) marcas de dientes, ocasionadas por interacciones sociales con otros delfines, y b) despigmentación que lo hace útil para su identificación individual. 


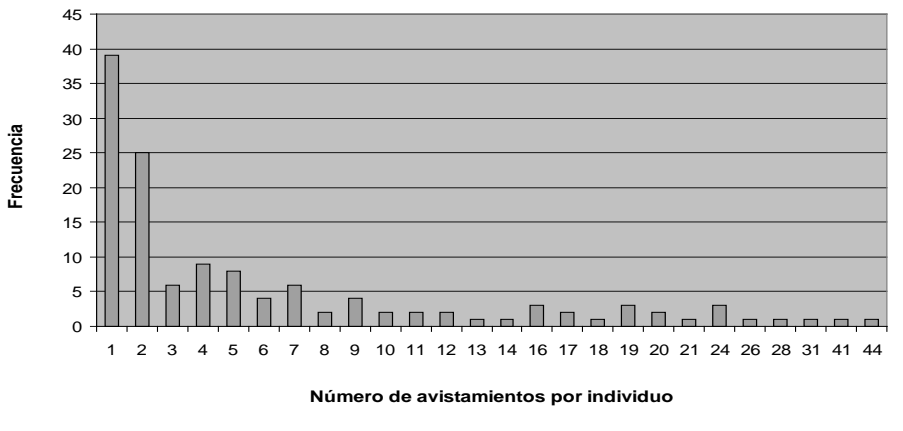

Figura 3. Frecuencia del número de re-avistamientos por individuo de T. truncatus en la Isla Tenerife (Islas Canarias), España en el 2005 y 2006.

\section{Composición del grupo.}

La presencia de crías y el tamaño grupal se realizó por observación directa en la embarcación a la hora del avistamiento. Las crías fueron identifican por tres características típicas, eran más pequeñas que el resto de delfines, por la coloración gris más claro y siempre se encontraron nadando junto a sus madres o libres, pero siempre dentro del grupo. Los delfines fueron clasificados como juveniles o inmaduros $(<2,5 \mathrm{~m})$, menos robustos, usualmente con marcas menos diferenciadas o con ausencia de las mismas en la aleta dorsal, y adultos (> 2,5 m) más robustos con marcas diferenciadas en la aleta dorsal (Díaz et al., 2005).

Se utilizó el programa SPSS (Statistical Package for the Social Sciences) 15,0 para identificar la ecuación de regresión que se ajuste adecuadamente a los datos obtenidos de frecuencia de animales "recapturados” y de frecuencia de tamaño grupal. Patrones de asociación.

Para calcular los coeficientes de asociación (CA) entre delfines que se han avistado juntos repetidas veces se empleó el programa SOCPRO 1,3 analizado en MATLAB (The Math Works, Inc., Natick., Mass., EUA, 1999) (Whitehead, 1999). Se utilizó el índice simple de HWI (Half Weight Index) para determinar la asociación entre individuos:

$$
\text { HWI }=\frac{X}{X+1 / 2\left(Y_{a}+Y_{b}\right)}
$$

Tabla 1. Seis ecuaciones de regresión para determinar la relación entre el número de avistamientos por individuo de T. truncatus y su frecuencia en la isla Tenerife (Islas Canarias), España en el 2005 y 2006.

\begin{tabular}{lccccccccc}
\hline \multirow{2}{*}{ Ecuación } & \multicolumn{4}{c}{ Modelo Resumen } & \multicolumn{3}{c}{ Parámetros estimados } \\
\cline { 2 - 10 } & $\mathrm{R}^{2}$ & $\mathrm{~F}$ & $\mathrm{gl}_{1}$ & $\mathrm{gl}_{2}$ & Sig. & Constante & $\mathrm{b}_{1}$ & $\mathrm{~b}_{2}$ & $\mathrm{~b}_{3}$ \\
\hline Lineal & 0,35 & 10,94 & 1 & 20 & 0,004 & 14,67 & $-0,73$ & & \\
Cuadrática & 0,64 & 17,21 & 2 & 19 & $<0,001$ & 25,74 & $-3,22$ & 0,09 & \\
Cúbica & 0,82 & 27,31 & 3 & 18 & $<0,001$ & 37,10 & $-7,91$ & 0,52 & $-0,01$ \\
Potencia & 0,77 & 70,18 & 1 & 20 & $<0,001$ & 32,12 & $-1,04$ & & \\
Crecimiento & 0,55 & 24,65 & 1 & 20 & $<0,001$ & 2,37 & $-0,10$ & \\
Exponencial & 0,55 & 24,65 & 1 & 20 & $<0,001$ & 10,74 & $-0,10$ & \\
\hline
\end{tabular}

Variable Dependiente: frecuencia. Variable Independiente: avistamientos. $\mathrm{R}^{2}$ : coeficiente de determinación. F: estadístico de Fisher. $\mathrm{gl}_{1}$ y $\mathrm{gl}_{2}$ : grados de libertad. Sig.: significancia $\mathrm{o}$ probabilidad. Const.: constante. $b_{1}, b_{2}$ y $b_{3}$ : coeficientes de la ecuación de regresión.
Donde, $\mathrm{X}$ es el número de veces en el que ambos individuos (a y b) fueron avistados juntos en el mismo grupo, $Y_{a}$ número de veces en el que el individuo a fue avistado e $\mathrm{Y}_{\mathrm{b}}$ número de veces en el que el individuo b fue avistado (Eisfeld, 2003; Robinette et al., 2003; White \& Smith, 2007). Este índice varía entre 0,00 para dos delfines que nunca han sido visto juntos, a 1,00 para dos delfines que siempre han sido avistados juntos (Herzing \& Brunnick, 1997). Se empleó el HWI en esta investigación debido al contexto social de análisis de cetáceos depredadores marinos y al ser el método más simple de asociación empleado (Robinette et al., 2003; Bouveroux \& Mallefet, 2010; Santos, 2010).

\section{Resultados.}

Del total de avistamientos o salidas realizados en el 2005 y en el 2006 fueron utilizados 287 (73,4\%) de 391. En ambos años se llevaron a cabo un total de 161 días de avistamientos, en el 2005 (59\%) y en el 2006 (41\%). Un total de 129 delfines adultos fueron fotoidentificados (97 en el 2005 y 102 en el 2006). La gran mayoría de los delfines foto-identificados se observó menos de cinco veces, con uno de los delfines siendo observado hasta 44 veces (Figura 3). El promedio mensual fue de 33 delfines nariz de botella en el 2005 y 25,4 delfines en el 2006.

De los 129 delfines identificados, 70 (54,3\%) se han re-avistados en el 2006, lo que nos indica que un aproximado al $50 \%$ de los delfines identificados son residentes. De los delfines residentes, los que se observaron con mayor frecuencia fueron los siguientes: uno que fue avistado en 13 meses $(72,2 \%)$, otros tres delfines fueron re-avistados en 12 meses (66,6\%) y también cuatro delfines fueron avistados en 11 meses (61,11\%). Mientras que en el 2005 se identificaron 97 individuos, en el 2006 se identificaron otros 32 individuos nuevos para el catálogo. Los resultados para los índices de similaridad entre los delfines avistados el año 2005 y el año 2006 muestran para $\mathrm{I}_{\mathrm{J}}, \mathrm{I}_{\mathrm{S}}$ y $\mathrm{I}_{\mathrm{O}-\mathrm{B}}$ valores de 0,51, 0,68 y 0,71, respectivamente. Estos tres índices muestran más del 50\% de similaridad en relación a los delfines foto-identificados entre el 2005 y el 2006.

Se registraron grupos que variaron en el número de individuos, desde 2 delfines hasta grupos de aproximadamente 50 delfines (Figura 4), pero lo más común fueron los avistamientos de 
grupos no muy numerosos, de entre 2 a 10 individuos. Un promedio de 7,47 delfines por grupo fue observado en esta población costera. Siendo en la frecuencia de distribución, la moda de 4 delfines por grupo (Figura 4).

En el caso de la frecuencia $(\mathrm{X})$ del número de delfines avistados por individuo y por grupo (Y), de las seis ecuaciones de regresión, la que mejor explica la relación entre ambas variables es la ecuación cúbica en ambos casos (Figura 4), debido a que presentó el $\mathrm{r}^{2}$ más alto (Tablas 1 y 2).

El tamañ1o poblacional con base al estimador de Petersen es de 143,44 delfines, en un área de 180 km², delfines se avistaron juntos cada par entre sí seis veces durante los dos años $(C A=0,29$ y $C A=0,30$, respectivamente). Los índices de asociación HWI variaron entre los delfines en la Isla Tenerife (España) entre 0,09 a 0,30 .

Un total de 14 delfines se avistaron junto con crías (10,9\%). Los avistamientos de crías fueron en junio 2005 y en julio 2006 (Tabla 3). El 31 de agosto del 2005 se tomó una fotografía de dos delfines en actitud de apareamiento, por lo que se podría indicarse que en esta zona y época se aparea $T$. truncatus. Otra evidencia, es que el mes donde se avistaron más crías fue en agosto, y los delfines tienen un periodo de gestación de 1 año. La etapa probable de reproducción del delfín nariz de botella para la isla Tenerife es en el verano (junio a noviembre).

Los delfines que más veces se han reavistado, son los que tuvieron marcas más definidas o fáciles de identificar (Figura 5). Un delfín se re-avistó hasta 44 veces, lo cual más al norte que las embarcaciones turísticas alcanzan, hasta "La Arenita” el punto más al sur. La densidad de delfines en el área de estudio fue de 0,79 delfines por $\mathrm{km}^{2}$.

En el caso de la estructura poblacional de los 287 avistamientos que se han realizado en ambos años, el $44,7 \%$ de los grupos están compuestos por grupos de solamente adultos, el $34,5 \%$ esta compuesto por adultos y crías, el 20,8\% por grupos de adultos, juveniles y crías. De los 129 delfines identificados durante los dos años del estudio, se confirma que existen dos hembras, debido que se las ha visto más de 4 veces junto a una cría, porque han sido avistadas junto con sus crías 11 y 8 veces, respectivamente. Se ha observado cierto grado de asociación de una hembra con otra, debido a que se han avistado repetidamente juntas $(C A=0,09)$. Se ha observado una baja asociación entre algunos delfines, por ejemplo dos delfines hembras se avistaron juntos 10 veces (CA $=0,24)$, otros dos delfines se avistaron juntos ocho veces durante ambos años $(C A=0,23)$, dos pares de

Tabla 3. Número de crías y número de avistamientos con crías de T. truncatus en la isla Tenerife (Islas Canarias), España en el 2005 y 2006.

\begin{tabular}{ccccccccccccc}
\hline & ene & feb & mar & Abr & may & jun & Jul & ago & sep & oct & nov & dic \\
\hline 2005 & SD & SD & SD & SD & X & $1^{*}(1)$ & $3(8)^{* *}$ & $5(9)$ & $3(3)$ & $1(1)$ & $1(1)$ & $2(2)$ \\
2006 & X & X & X & X & SD & X & $1(1)$ & $2(6)$ & $1(1)$ & $1(1)$ & $2(2)$ & SD \\
\hline
\end{tabular}

$\mathrm{NE}=$ no evaluados. $\mathrm{X}=$ sin crías. ${ }^{*}=$ número de crías avistadas. ${ }^{* *}=$ número de avistamientos con crías entre paréntesis. puede ser debido a que este delfín en particular tiene una aleta dorsal muy deformada y es fácil de identificar incluso desde muy lejos. Este delfín en particular durante los 2 años de toma de fotografías nos dio una clara evidencia del cambio que las heridas pueden sufrir a lo largo del tiempo (Figura 2).

\section{Discusión.}

Varios factores físicos, químicos y biológicos influyen en la presencia del delfín nariz de botella (residencia, tamaño poblacional, composición del grupo y patrones de asociación) en un espacio y tiempo determinado (Pérez-Cao et al., 2009). De los en el 2006 fueron identificados como residentes (Martínez-Serrano et al., 2011), debido a que se observaron en ambos periodos de estudio. Esto pudiera deberse a la disponibilidad de alimento en la zona (Acevedo \& Wúrsig, 1991; Díaz, 2006). PérezCao et al. (2009) señalan que el delfín nariz de botella es un consumidor oportunista, y que el alimento es un factor clave en la fidelidad de residencia a estudio existen tres piscigranjas tipo jaulas flotantes, para el cultivo de Sparus aurata Linnaeus $1758 \quad \mathrm{y}$ Dicentrarchus labrax 129 delfines identificados, 54,3\% al ser re-avistados un área. En la zona de 


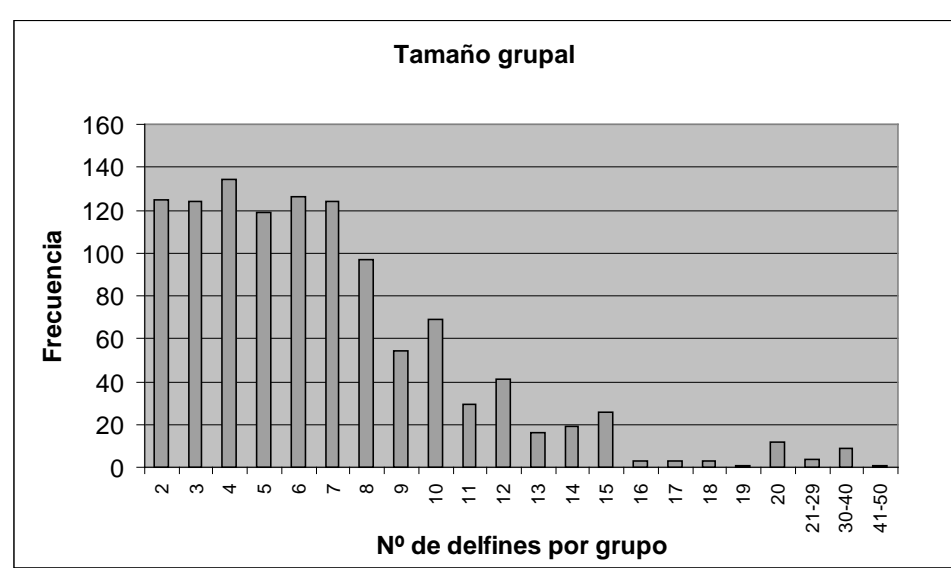

Figura 4. Frecuencia del número de delfines avistados de $T$. truncatus por grupo en la Isla Tenerife (Islas Canarias), España en el 2005 y 2006.

Linnaeus 1758, comúnmente llamados “dorada” y "lubina”, respectivamente (Díaz, 2006). Un gran número de avistamientos se realizaron en los alrededores de estos criaderos, así una parte de la comida balanceada posiblemente atravesó las mallas y atrajo otras especies de peces más pequeños del género Mugil, Salpa y Sardina, que son atraídos por las jaulas flotantes y que son el alimento de los delfines (Bearzi et al., 2008). Los delfines nariz de botella aprovechan a los peces del criadero que se escapan de las jaulas y cazan en los alrededores de los criaderos (Díaz et al., 2005). Se han identificado delfines que solo han sido foto-identificados una sola vez durante los dos años de estudio lo que significa que la zona de estudio sería solo una parte del territorio de otros grupos poblacionales de delfines nariz de botella, como lo señalan otros trabajos realizados en Texas, USA (Irwin \& Würsig, 2004) y en el estuario de Shannon, Irlanda (Rogan et al., 2000). El valor de residencia calculado en este trabajo es comparable con Fury \& Harrison (2008), quienes señalan valores de $37 \%$ y $60 \%$ para los delfines residentes en Australia. Pero ligeramente más alto al observado en México de 12\% y Belice de 30\% (Kerr et al., 2005; Martínez-Serrano et al., 2011). En otra especie de delfín Sotalia guianensis (Gervais, 1853), en Brasil se ha visto que la mayoría no son residentes (93\%) y con baja fidelidad en el área (Rossi-Santos et al., 2007).

El delfín nariz de botella de Tenerife forma asociaciones de larga duración (Connor et al., 1999; Eisfeld, 2003; Graves, 2008). Existen dos tipos de agrupaciones en los delfines nariz de botella, la agrupación primaria o "pod", que consta de pocos individuos (2-10 individuos) (Kerr et al., 2005) у donde estos siempre se avistan juntos y realizan actividades juntos como grupo como la alimentación, y también existe los grupos secundarios o manadas que es la unión de varias de las agrupaciones primarias, conformando así grupos de hasta 100 individuos de carácter temporal (Shane et al., 1982). En el presente estudio el número de delfines que forman grupo varió mayormente entre 2 a 10 individuos (Figura 4).

La conformación de los grupos de delfines ha sido mayormente de adultos (44,7\%). Siendo muy común encontrar grupos de 2 a 5 delfines adultos. Las agrupaciones más numerosas, pero no comúnmente observadas son las que tenían entre 10 y 15 delfines, donde se encontraron la mayor cantidad de crías y juveniles. Estos resultados son similares a un estudio realizado en Australia, donde los grupos que presentaban las crías eran ligeramente más grandes que los que no la tenían (Félix, 1997; Möller et al., 2002).

Los delfines nariz de botella forman agrupaciones muy variadas en tamaño: grupos grandes que se encuentran en aguas profundas y alejadas de la costa, y grupos más pequeños cerca a las costas marinas (Martínez-Serrano et al., 2011). Esta investigación tiene características de grupos pequeños de ambientes cerrados con 3 a 7 individuos comunes en diferentes localidades (Morteo et al. 2004; Martínez-Serrano et al., 2011). Lodi \& Monteiro-Neto (2012) muestra en T. truncatus en Brasil valores promedio de 13,7 $\pm 7,1$ individuos, pero con tendencia a la disminución entre años y correlacionándolos con la calidad del hábitat. En Belice, América Central los grupo fueron más bajos de 2,9 $\pm 2,3$, y en Cuba, el grupo más frecuente es ocho (Kerr et al., 2005; Pérez-Cao et al., 2009). En el delfín $S$. guianensis en un estuario de Brasil se han registrado grupos de 3,7 $\pm 2,6$ individuos (Santos, 2010).

Félix (1994) en el Golfo de Guayaquil (Ecuador), Rogan et al. (2000) en el estuario de Shannon (Irlanda), Irwin \& Würsig (2004) en las aguas de Texas (USA) y Pérez-Cao et al. (2009) en Matanzas (Cuba) encontraron una densidad de delfines $\mathrm{km}^{2}$ de 0,89, 0,94, 1,01 y 1,28, respectivamente. En las costas de la Isla Tenerife la densidad de delfines fue 0,79 delfines $\cdot \mathrm{km}^{2}$.

Durante los meses de junio hasta diciembre se observaron crías, pero el mes donde se observó el número más elevado de crías, fue agosto; esto concuerda con otras investigaciones en otras localidades de los delfines nariz de botella en el estuario de Shannon (Irlanda) (Rogan et al., 2000; Eisfeld, 2003).

Se confirma la existencia de alianzas entre individuos, los cuales se observaron más de cuatro veces juntos durante los dos años del estudio, dos delfines se han avistado juntos 10 veces y se puede confirmar que uno de ellos es una hembra por que se le ha avistado repetidamente con una cría nadando al 
costado. Otro indicio de asociación entre hembras es la de dos delfines que se avistaron juntos tres veces, debido a que han sido avistadas con crías varias veces durante los dos años de estudio, por lo que se puede corroborar así la teoría de que hay cierta asociación entre hembras con la misma madurez sexual (Felix,
1997; Eisfeld \& Robinson, 2004). Bouverou \& Mallafet (2010) han observado fuertes asociaciones entre pares o tríos de machos del delfín nariz de botella en Florida, EEUU, presentando las hembras más bajas asociaciones que los machos.

Los índices de asociación HWI variaron entre los

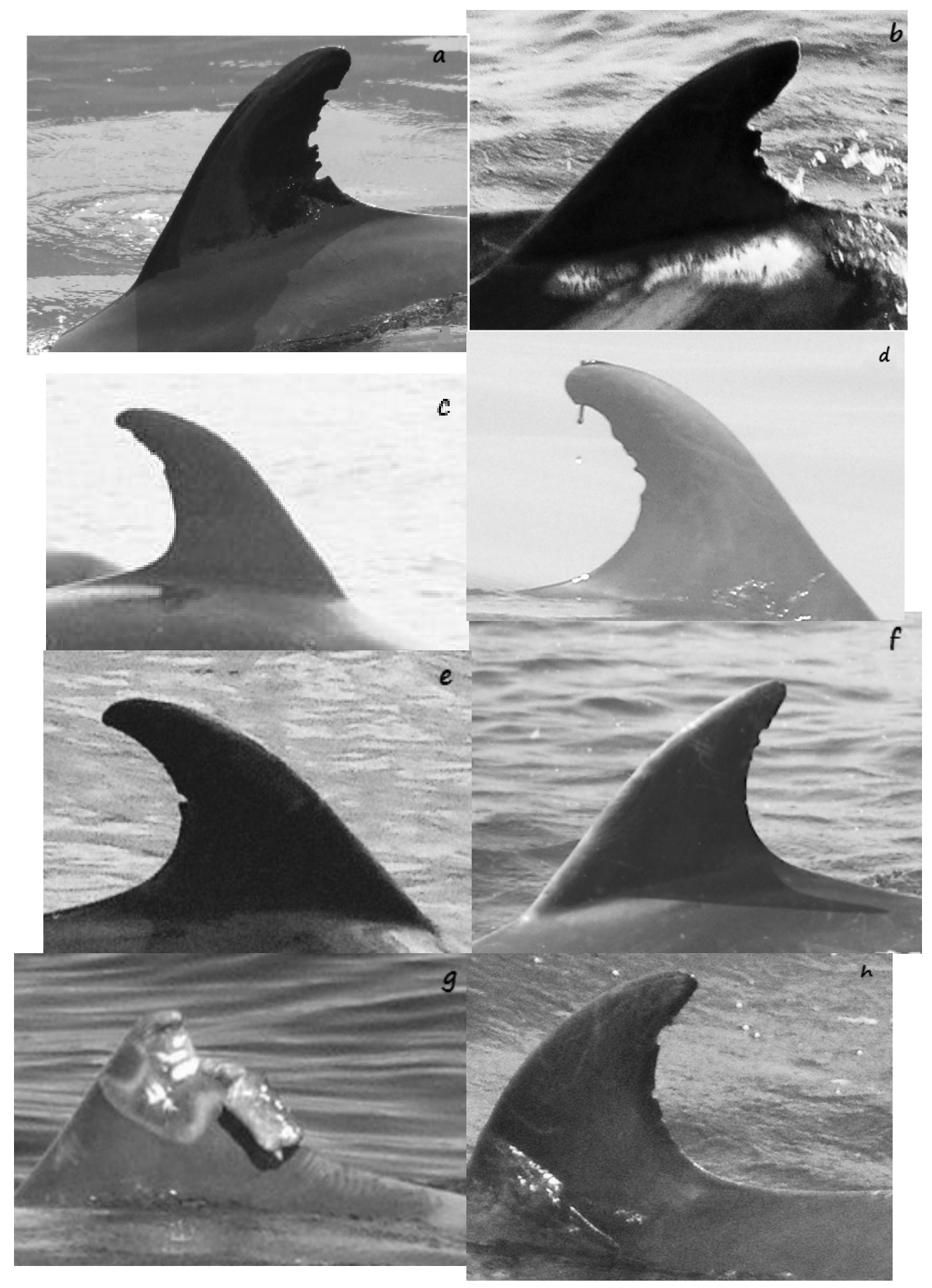

Figura 5. Secuencia de fotografías del cambio progresivo de la herida de uno de los delfines en la aleta dorsal. a. 19.07.05. b. 16.08.05. c. 16.11.05. d. 30.11.05. 
delfines en la Isla Tenerife (España) entre 0,09 a 0,30. Estos valores fueron más altos a lo observado en Florida, EEUU de 0,11 (Bourevoux \& Mallefet, 2010).

Los grupos de lo delfines son fluidos, los delfines ingresan y salen constantemente, se considera que los grupos generalmente están compuestos por hembras jóvenes y adultas con sus crías, generalmente no se encuentran machos dentro de estos grupos (Shane et al., 1982).

\section{Conclusiones.}

Se foto-identificaron en la isla de Tenerife (Islas Canarias), España, 129 delfines nariz de botella $T$. truncatus, considerados como residentes 70 (54,3\%). El número de delfines que conforman los grupos en la mayoría varió entre 2 a 10 individuos, pero se avistaron grupos compuestos por más de 50. La población estimada en el área de estudio fue de 143 delfines nariz de botella, con una densidad de 0,79 delfines $\mathrm{km}^{-2}$. Los meses de presencia de crías fueron junio a noviembre, con el pico en agosto (verano). El índice de asociación (HWI) mostró valores entre 0,09 y 0,30 .

\section{Literatura citada.}

Acevedo A. \& Wúrsig W. 1991. Preliminary observations on bottlenose dolphins, Tursiops truncatus, at Isla del Coco, Costa Rica. Aquatic Mammals. 17:148-151.

Aguayo-Lobo A. 1999. Los cetáceos y sus perspectivas de conservación. Estudios Oceanológicos. 18: 35-43.

ACS (American Cetacean Society). 2007. American Cetacean Society Fact Sheet. [citado 11 ene 2011] Disponible en: URL: http://www.acsonline.org/factpack/btlnose.htm.

Bearzi M. 2005. Aspects of the ecology and behaviour of bottlenose dolphins (Tursiops truncatus) in Santa Monica Bay, California. Journal of Cetacean Research and Management. 7: 75-83.

Bearzi G., Fortuna C. \& Reeves R. 2008. Ecology and conservation of common bottlenose dolphins Tursiops truncatus in the Mediterranean Sea. Mammal Review. 39: 92-123.

Bejder L., Samuels A., Whitehead H., Gales N., Mann J., Connor R., Heithaus M.R., Watson-Capps J., Flaherty C. \& Krutzen M. 2006. Decline in relative abundante of bottlenose dolphins exponed to long-term disturbance. Conservation Biology. 20: 1791-1798.

Bouverox, Th. \& Mallafet, J. 2010. Social structure of bottlenose dolphins, Tursiops truncatus, in Panama City, Florida. Journal of the Marine Biological Association of the United Kingdom. 90: 1685-1692.

Carballo M., Arbelo M., Esperón F., Méndez M., De La Torre A. \& Muñoz M.J. 2008. Organochlorine residues in the blubbler and liver of bottlenose dolphins (Tursiops truncatus) stranded in the Canary islands, North Atlantic Ocean. Environmental Toxicology. 23: 200-210.

Carrillo M., Pérez-Vallaza C. \& Álvarez-Vásquez R. 2010 Cetacean diversity and distribution off Tenerife (Canary Islands). Marine Biodiversity Records. 3: e97 (9 pages).
CC (Canarias Conservación). 2010. [citado 20 dic 2010]. Disponible en: URL: http://www.canariasconservacion.org/CETCANCaldero n.htm.

CITES (Convención sobre el comercio Internacional de especies amenazadas de fauna y flora silvestre). 2007. Disponible en: URL: Species Database. [citado 16 feb 2007]. Disponible en: URL: http://www.cites.org/eng/resources/species.html.

Connor C., Heithaus M. \& Barre L.M. 1999. Superalliance of bottlenose dolphins. Nature. 397: 571-572.

Díaz B., Marini L. \& Polo F. 2005. The impact of a fish farm on a bottlenose population on the Mediterranean sea. Thalassas. 21: 65-70.

Díaz, B. 2006. Bottlenose dolphin (Tursiops truncatus) predation on a marine fin fish farm: some underwater observations. Aquatic Mammals. 32: 305-310.

Eisfeld S. \& Robinson K. 2004. The Sociality of bottlenose dolphins in the outer Southern Moray Firth, NE Scotland: Implications for current management proposals?. European Research on Cetaceans. 18 (on CD-ROM).

Eisfeld S.M. 2003. The social affiliation and group composition of bottlenose dolphins (Tursiops truncatus) in the outer southern Moray Firth, NE Scotland. Thesis submitted for the dregree of Master in Science. School of Biological Sciences. University of Wales, Bangor. 70 p.

Esperón F., Fernández A. \& Sánchez-Vizcaíno J.M. 2008. Herpes simplex-like infection in a bottlenose dolphin stranded in the Canary Island. Disease of Aquatic Organisms. 81: 73-78.

Felix F. 1994. Ecology of the coastal bottlenose dolphin Tursiops truncatus in the gulf of Guayaquil, Ecuador. Investigations on Cetacea. 25: 235-256.

Felix F. 1997. Organization and social structure of the coastal bottlenose dolphin Tursiops truncatus in the gulf of Guayaquil, Ecuador. Aquatic Mammals. 23: 1-16.

Foley A., Mcgrath D., Berrow S. \& Gerritsen H. 2010. Social structure within the bottlenose dolphin (Tursiops truncatus) populations in the Shannon estuary, Ireland. Aquatic Mammals. 36: 372-381.

Folkens P., Reeves R., Stewart B., Clapham P. \& Powell J. 2002. National Audubon Society: guide to Marine Mammals of the World. pp. 358-361. Alfred A. Knopf, Inc., New York, USA and Random House of Canada, Limited, Toronto, Canada.

Fury C.A. \& Harrison P.L. 2008. Abundance, site fidelity and range patterns of Indo-Pacific bottlenose dolphins (Tursiops aduncus) in two Australian subtropical estuaries. Marine and Freshwater Research. 59: 10151027.

Genov T., Kotnjek P., Lesjak J., Hace A. \& Fortuna C.M. 2008. Bottlenose dolphins (Tursiops truncatus) in Slovenian and adjacent waters (Northern Adriatic Sea). Annales, Series Historia Naturalis. 18: 227-244.

Graves V. 2008. Life history parameters and social associations of female bottlenose dolphins (Tursiops truncatus) off North Carolina, USA. Dissertation degree of Doctor of Philosophy. Department of Environment. Duke University. 163 p.

Grellier K., Hammond P., Wilson B., Sanders-Reed C. \& Thompson P. 2003. Use of photo-identification data to 
quantify mother-calf association patterns in bottlenose dolphins. Canadian Journal of Zoology. 81:1421-1427.

Herzing DL \& Brunnick BJ. 1997. Coefficients of association of reproductively active fermale Atlantic spotted dolphins, Stenella frontalis. Aquatic Mammals. 23: $155-162$

Irwin L \& Würsig B. 2004. A small resident community of bottlenose dolphins, Tursiops truncatus, in Texas: Monitoring Recommendations. Gulf of Mexico Science. 22: $13-21$.

Jaber J.R., Pérez J., Carballo M., Arbelo M., Espinosa De Los Monteros A., Herráez P., Muñoz J., Andrada M., Rodríguez F. \& Fernández A. 2005. Hepatosplenic large cell immunoblastic lymphoma in a bottlenose dolphin (Tursiops truncatus) with high levels of polychlorinated biphenyl congeners. Journal of Comparative Pathology.132:242-247.

Kerr, K.A., Defran, R.H. \& Campbell, G.S. 2005 Bottlenose dolphins (Tursiops truncatus) in the Drowned Cayes, Belize; group size, site fidelity and abundance. Caribbean Journal of Science. 41: 172-177.

Lodi L., Wedekin L.L., Rossi-Santos M.R. \& Marcondes M.C. 2008. Movements of the Bottlenose Dolphin (Tursiops truncatus) in the Rio de Janeiro State, Southeastern Brazil. Biota Neotropica. 8: 205-209.

Lodi, L. \& Monteiro-Neto, C. 2012. Group size and composition of Tursiops truncatus (Cetacea: Delphinidae) in a coastal insular habitat off southeastern Brazil. Biotemas. 25: 157-164.

Majluf P. \& Reyes J. 1989. The marine mammals of Perú: A Review in the Peruvian Upwelling ecosystem: Dynamics and interactions. pp. 344-363. ICLARM Conference Proceedings 18, 438 p. Pauly, D., Muck, P., Mendo, J. \& Tsukayama, L. (eds). Instituto del Mar del Perú (IMARPE), Callao, Perú; Deutsche Gesellschaft für Technische Zusammernarbeit (GTZ) GmbH, Eschborn, Federal Republic of Germany; e International Center for Living Aquatic Resources Management (ICLARM), Manila, Philippines.

Martinez-Serrano I., Serrano A., Heckel G. \& Schramm. 2011. Distribution and home range of bottlenose dolphins (Tursiops trunctatus) off Veracruz, Mexico. Ciencias Marinas. 37: 379-392.

May-Collado L.J., Agnarsson I., Palacios D., Taubitz E. \& Wartzok D. 2007. The status of the bottlenose dolphin (Tursiops Truncatus) population of Bocas del Toro, Panama: preliminary results based on a three year ongoing study. Fundacion Keto Internal Report IRLJMC-KETO01-BOCAS. 1-26 p.

Möller L.M., Allen S.J. \& Harcourt R.G. 2002. Group characteristics, site fidelity and seasonal abundance of bottlenose dolphins (Tursiops aduncus) in Jervis Bay and Port Stephens, Southeastern Australia. Australian Mammalogy. 24:11-21.

Moreno C. 2001. Métodos para medir la Biodiversidad. M\&T - Manuales y Tesis SEA. Cooperación Iberoamericana CYTED. UNESCO Orcyt. Sociedad Entomológica Aragonesa. $1^{\circ}$ Ed. México. 84 p.

Morteo E., Heckel G., Defran R.H. \& Schramm Y. 2004. Distribution, movements and group size of the bottlenose dolphin (Tursiops truncatus) to the south of San Quintín Bay, Baja California, Mexico. Ciencias Marinas. 30: 35-46.
Navarro A. 2002. Diversidad genética del DNA mitocondrial del delfín mular (Tursiops truncatus) y calderón tropical (Globicephala macrorhynchus) en el Archipiélago Canario. Folia Canariensis Academiae Scientiarum.14: 341-345.

Pérez-Cao, H., López, N., Blanco, M., Lio, V. \& GonzálesSansón, G. 2009. Abundancia y distribución del delfín (Tursiops truncatus, Montagu, 1821) en la costa norte de la provincia de Matanza, Cuba. Revista de Investigación Marina. 30: 55-61.

Pérez-Vallaza C., Álvarez-Vásquez R., Cardona L., Pintado C. \& Hernández-Brito J. 2008. Cetacean diversity of the west coast of La Palma (Canary Island). Journal of the Marine Biological Association of the UK. 88: 12891296.

Plasencia M., Rodriguez J.L, Herrera H., Delgado A. 2001. Observación de cetáceos en Canarias; apuntes para una nueva reglamentación. Galemys. 13: 107-118.

Randic, S., Connor, R.C., Sherwin, W.B. \& Krützen, M. 2012. A novel mammalian social structure in IndoPacific bottlenose dolphins (Tursiops sp.) complex male alliances in na open social network. Proceeding of Royal Society B Biological Science. 279: 3083-3090.

Robinette, H.A. R., Bentzen, P., Marsh, J. \& Ha, J.C. 2003. Kinship and association in social foraging Northwestern crows (Corvus caurinus). Bird Behaviour. 15: 65-75.

Rogan E., Ingram S., Holmes B. \& O’Flanagan C. 2000. A survey of bottlenose dolphins in the Shannon Estuary. Marine Resource Series, pp. 9. Unpublished report, Marine Institute, Shannon Dolphin and Wildlife Foundation, Kilrush, County Clare, Ireland. 49 pp. [citado 15 nov 2010]. Disponible en: URL: http// www.shannondolphins.ie.

Rossi-Santos, M.R., Wedekin, L.L. 6 Monteiro-Filho, E.L.A. 2007. Residence and site fidelity of Sotalia guianensis in the Caravelas river estuary, eastern Brazil. Journal of the Marine Biological Association of the United Kingdom. 87: 207-212.

Santos, M.C.O. 2010. Guiana dolphin (Sotalia guianensis) displaying beach hunting behavior in the Cananéia estuary, Brazil: Social context and conservation issues. Brazilian Journal of oceanography. 58: 143-152.

Shane S., Wells R., Wursig B. \& Odell D. 1982. Ecology, behavior and social organization of the bottlenose dolphin: a review. Marine Mammal Science. 2: 34-63.

Torres L.G., Mclellan W.A., Meagher E. \& Pabst D.A. 2005. Seasonal distribution and relative abundante of bottlenose dolphins, Tursiops truncatus, along the US mid-Atlantic Coast. Journal of Cetacean Research and Management. 7: 153-161.

Vásquez, C.L., Serrano, A. \& Galindo, J.A. 2009. Estudio preliminar sobre la biodiversidad, distribución y abundancia de cetáceos en aguas profundas del golfo de México. Revista UDO Agrícola. 9: 992-997.

Verme V. \& Iannacone J. 2011. Catálogo de fotoidentificación del delfín nariz de botella (Tursiops truncatus) en las Islas Canarias, España: una línea de base de información para su conservación. The Biologist (Lima). 9: 105-119.

Vonk R. \& Martel V.M. 1988. First list of odontocetes from the Canary island, 1980-1987. In European Research on cetaceans. Evans, PG.H. (Ed.). Lisboa, Portugal. pp. 3135 . 
Waerebeek K., Van Bressem M., Felix F., Alfaro-Shigueto J., Garcia A., Chávez L., Onton K., Montes D. \& Bello, R. 1997. Mortality of dolphins and porpoises in costal fisheries off Peru and southern Ecuador in 1994. Biological Conservation. 8: 43-49.

Warburton, C.A., Parsons E.C.M. \& Goodwin H. 2000 Marine wildlife tourism and whale-watching on the Isle of Mull, Scotland. Paper presented to the scientific committee at the $52^{\text {nd }}$ meeting of the International
Whaling Comission. 11-28 June, Australia. SC/52/WW17. 20 p.

WDCS (Whale and Dolphin Conservation Society).2007. Bottlenose dolphins in captivity. [citado 4 de Ene 2007]. Disponible en: URL: http://www.wdcs.org.

White, D.J. \& Smith, A. 2007. Testing measures of animal social association by computer simulation. Behaviour. 144: $1447-1468$.

Whitehead H. 1999. Testing association patterns of social animals. Animal Behavior Forum. 57: F26-F29.

1 Museo de Historia Natural (MHN)-Universidad Ricardo Palma (URP). Av.Benavides 5440, Santiago de Surco, Lima, Perú.

2 Laboratorio de Ecofisiología Animal, Facultad de Ciencias Naturales y Matemática, Universidad Nacional Federico Villarreal (UNFV), Av. Río Chepén s/n, El Agustino, Lima, Perú. Correo electrónico: joseiannacone@gmail.com 